\title{
A Comparative Study of the Optimum Tuned Mass Damper for High-rise Structures Considering Soil-structure Interaction
}

\author{
Ali Kaveh ${ }^{1 *}$, Shaylin Rezazadeh Ardebili ${ }^{1}$ \\ ${ }^{1}$ School of Civil Engineering, Iran University of Science and Technology, 16846-13114 Tehran, Iran \\ * Corresponding author, e-mail: alikaveh@iust.ac.ir
}

Received: 19 April 2021, Accepted: 28 May 2021, Published online: 02 June 2021

\begin{abstract}
The present paper focuses on the optimum design of tuned mass damper (TMD) as a device for control of the structures. The optimum free vibration parameters such as period and damping ratio depend on the soil condition. For this reason, the seven meta-heuristic algorithms namely colliding bodies optimization (CBO), enhanced colliding bodies optimization (ECBO), water strider algorithm (WSA), dynamic water strider algorithm (DWSA), ray optimization (RO) algorithm, teaching-learning-based optimization (TLBO) algorithm and plasma generation optimization (PGO) are used to find the TMD parameters considering soil-structure interaction (SSI) effects. These optimization methods are applied to a benchmark 40-story structure. For comparison, the obtained results of these algorithms are compared. The capability and robustness of the algorithms are investigated through the benchmark problem. The results are shown that the soil type affects the optimum values of the TMD parameters, especially for the soft soil. To evaluate the performance of the obtained parameters in both the frequency and time domains, time history displacement and acceleration transfer function of the top story of the structure are calculated for the model with and without considering the SSI effects.
\end{abstract}

Keywords

meta-heuristic optimization algorithms, structural control, tuned mass dampers, soil-structure interaction

\section{Introduction}

Civil engineering structures can be damaged by undesired vibrations due to winds and earthquakes. Different strategies of structural control are used to decrease these vibrations. One of the most economical control strategies is passive control devices. A tuned mass damper (TMD) is invented as a vibration absorber device. This is one of the simplest and reliable devices consisting of mass, stiffness, and damping members. The performance of TMDs extremely depends on the parameters of mass, stiffness, and damping. Therefore, the optimum design of the TMD parameters is an optimization problem that has been widely investigated.

Many researchers have presented optimal tuning of parameters. The closed-form relationships are utilized for designing the TMD system; however, these solutions are complex and multi-objective problems. Thus, finding a general solution will be difficult for investigation. In addition to closed-form solutions, meta-heuristic algorithms have been recently utilized to find optimal TMD parameters. Meta-heuristics are nature-inspired techniques for optimization problems [1]. They are more flexible and simple than mathematical methods. Due to the characteristics of these methods, many researchers have utilized these methods to find optimum or near-optimum solutions to the problems. These advantages have encouraged designers to use meta-heuristic algorithms to solve different problems such as the optimum design of structures, optimum control, optimum design of the TMD system, etc. [2]. Hadi and Arfiadi [3] proposed a methodology based on GA algorithm for optimum design of the TMD system used shear building structures subjected to earthquake excitation. Other meta-heuristic algorithms such as particle swarm optimization (PSO) [4], bionic algorithm (BA) [5], harmony search (HA) [6], ant colony optimization (ACO) [7], charged system search (CSS) [8], colliding bodies optimization (CBO) [9] have been applied to the optimum tuning of parameters of the mass dampers.

The mentioned studies concern the optimum design of fixed-base structures. It should be noted that the effect of a flexible base in the tuning of the TMD system can be significant and changed the efficiency of these devices. 
The soil-structure interaction (SSI) effects would significantly change the dynamic responses and specification of structures such as natural frequencies, damping ratios, mode shapes, and tuning parameters of the TMD system [10].

The solutions of the optimization process can be obtained by frequency or time domain analyses. Time domain analysis of structures was performed in some of the studies such as Farshidianfar and Soheili [7] obtained the optimum values of TMDs including SSI effects using ant colony optimization (ACO) and the maximum acceleration plus 10 times the maximum displacement of stories was selected as the objective function. Also, the Artificial Bee Colony (ABC) [11] and shuffled complex evolution (SCE) [12] methods were implemented for the 40-story and 15-story structures for different earthquakes. Bekdaş and Nigdeli [13] researched the optimum design variables with limitations of the scaled stroke of TMD as the design constraint by two meta-heuristic algorithms including harmony search (HS) algorithm and bat algorithm (BA). Salvi et al. [14] contributed to investigate the effectiveness of an optimum TMD in decreasing the structural response of the low- and high-rise shear building structures. Etedali et al. [15] compared the performance of classical TMD and Friction tuned mass damper (FTMD) for tall buildings under earthquake excitation by multi-objective cuckoo search (MOCS) algorithm.

Another case of TMD for controlling the structures is multiple tuned mass dampers (MTMD). Li and Liu [16] examined the influence of the dominant frequency of ground motion on the optimum values and compared the performance of MTMD with the same stiffness and damping coefficient but with different masses. The effectiveness of the MTMD system on the control of irregular structures is investigated [17]. Li and Han [18] investigated the effects of the dominant ground frequency and soil characteristics on the performance of the MTMD. To increase the traditional TMD effects, tuned mass damper inerter (TMDI) was investigated to control a 10-story shear building benchmark [19]. The performance and robustness of the optimum TMDI under 25 far-fault (FF) ground motions were evaluated in the time domain with 12 different performance criteria and three different objective functions [20].

Due to the random nature of the earthquakes, the frequency domain analysis of structures should be used in tuning the TMD system rather than the time domain. Since earthquakes consist of various components with different frequencies, frequency domain analysis can be effective to find a steady stead response. In some investigations, the maximum amplitude of the transfer function of the structure is selected as an objective function. The teaching learning-based optimization (TLBO), flower pollination algorithm (FPA), harmony search (HS) [13], and colliding bodies optimization (CBO) algorithm [21] were applied to determine optimum parameters of the TMD system for fixed base structures.

In this study, the performance of seven different population-based meta-heuristic algorithms is studied in the optimum design of TMD for a 40-story benchmark structure. This study aims at providing the optimum design of TMD for a 40-story benchmark structure. The infinity norm of the acceleration transfer function of the top story is considered an objective function. Therefore, the optimum parameters of TMD are independent of the external excitation frequency. To find optimum parameters, different algorithms including colliding bodies optimization (CBO), enhanced colliding bodies optimization (ECBO), water strider algorithm (WSA), dynamic water strider algorithm (DWSA) ray optimization $(\mathrm{RO})$ algorithm, teaching-learning-based optimization (TLBO) algorithm, and plasma generation optimization (PGO) are employed. The convergence, accuracy, and computational effort of these algorithms are compared among themselves. The performance of the SSI system is evaluated in time and frequency domains. The effects of the different soil types and the TMDs with different masses are investigated on the optimum results.

The rest of this paper is organized as follows. The mathematical model and formulations for high-rise buildings considering SSI in Section 2 are provided. The optimization process is illustrated in Section 3 and the pseudo-code of applied algorithms in the present study is briefly introduced. These algorithms are used in a numerical example and the optimum values of the TMD tuning parameters are provided in Section 4. The performance of optimum parameters is discussed in the time and frequency domain in Section 5. Finally, the conclusions and some suggestions are outlined in Section 6.

\section{Mathematical models for high-rise buildings considering SSI}

A TMD as an additional SDOF is installed on the top of the structure. A convenient mathematical model is presented for the N-story shear building considering SSI shown in Fig. 1. The equation of motion for the structural system under earthquake excitation ( is given in matrix form [22]. 


$$
[M] \ddot{\boldsymbol{x}}(t)+[C] \dot{\boldsymbol{x}}(t)+[K] \boldsymbol{x}(t)=-\left[m^{*}\right] \ddot{x}_{g},
$$

where $[M],[C],[K]$, and $\left[m^{*}\right]$ are the mass, damping, stiffness, and acceleration mass matrices of the system defined in Eqs. (2)-(9), respectively; $\ddot{\boldsymbol{x}}(t), \dot{\boldsymbol{x}}(t)$ and $\boldsymbol{x}(t)$ are acceleration, velocity, and displacement vectors of the structural system. The vector $\boldsymbol{x}(t)$ for $(N+3)$ degree-of-freedom system will be defined as Eq. (10) where $x_{i}$ is the displacement of $i^{\text {th }}$ story and $x_{0}$ and $\theta_{0}$ are displacement and rotation of the base.

$$
[M]=\left[\begin{array}{ccc}
{\left[M_{f}\right]} & {\left[M_{v}\right]} & {[M Z]} \\
\vdots & m_{0}+\left(\sum_{i=1}^{N} m_{i}\right)+m_{d} & \left(\sum_{i=1}^{N} m_{i} z_{i}\right)+m_{d} z_{N} \\
\operatorname{sym} & \ldots & \left(\sum_{i=1}^{N} m_{i} z_{i}^{2}\right)+m_{d} z_{N}^{2}+I_{0}+\sum_{i=1}^{N} I_{i}
\end{array}\right]
$$$$
\left[M_{v}\right]=\left[\begin{array}{l}
m_{1} \\
m_{2} \\
\vdots \\
m_{N-1} \\
m_{N} \\
m_{d}
\end{array}\right]
$$

$$
[M Z]=\left[\begin{array}{l}
m_{1} z_{1} \\
m_{2} z_{2} \\
\vdots \\
m_{N-1} z_{N-1} \\
m_{N} z_{N} \\
m_{d} z_{N}
\end{array}\right]
$$

$$
[C]=\left[\begin{array}{ccccc} 
& & & 0 & 0 \\
& & & & \\
& & & \vdots & \vdots \\
0 & \cdots & 0 & c_{s} & 0 \\
0 & \cdots & 0 & 0 & c_{r}
\end{array}\right]
$$

$$
\left[C_{f}\right]=\left[\begin{array}{ccccc} 
& & & & \\
\left(c_{1}+c_{2}\right) & -c_{2} & & 0 & \\
-c_{2} & \left(c_{2}+c_{3}\right) & & \vdots & \\
& & \ddots & 0 & 0 \\
\operatorname{sym} & \ldots & -c_{N} & \left(c_{N}+c_{d}\right) & -c_{d} \\
& & 0 & -c_{d} & c_{d}
\end{array}\right]
$$

$$
[K]=\left[\begin{array}{ccccc} 
& & & 0 & 0 \\
& & & & \\
& & & 0 & 0 \\
0 & \cdots & 0 & k_{s} & 0 \\
0 & \cdots & 0 & 0 & k_{r}
\end{array}\right]
$$

$$
\left[K_{f}\right]=\left[\begin{array}{ccccc}
\left(k_{1}+k_{2}\right) & -k_{2} & & 0 & \\
-k_{2} & \left(k_{2}+k_{3}\right) & \ddots & \vdots & \\
& & \ddots & 0 & 0 \\
s y m & \ldots & -k_{N} & \left(k_{N}+k_{d}\right) & -k_{d} \\
& & 0 & -k_{d} & k_{d}
\end{array}\right]
$$

$$
\left[m^{*}\right]=\left[\begin{array}{c}
m_{1} \\
m_{2} \\
\vdots \\
m_{N-1} \\
m_{N} \\
m_{d} \\
m_{0}+\left(\sum_{i=1}^{N} m_{i}\right)+m_{d} \\
\left(\sum_{i=1}^{N} m_{i} z_{i}\right)+m_{d} z_{N}
\end{array}\right]
$$

$$
x(t)=\left[\begin{array}{c}
x_{1} \\
x_{1} \\
\vdots \\
x_{N-1} \\
x_{N} \\
x_{d} \\
x_{0} \\
\theta_{0}
\end{array}\right]
$$

Where $\left[M_{f}\right],\left[K_{f}\right]$ and $\left[C_{f}\right]$ are the matrices of mass, stiffness, and damping of a fixed based structure with a TMD placed on the top story; $m_{i}, I_{i}, k_{i}, c_{i}$, and $z_{i}$ are the mass, moment of inertia, stiffness, damping, and location of the $i^{\text {th }}$ story, respectively; $m_{o}$ and $I_{o}$ are the mass and moment of inertia of foundation; $m_{d}, c_{d}$, and $k_{d}$ are the mass, damping and stiffness coefficients of TMD. A rigid circular foundation on the ground surface is adopted in this study. The tall building is supported by this foundation with the swaying and the rocking dashpots, and the corresponding springs. The damping and stiffness coefficients of the swaying and rocking motion of soil depended 
on Poisson's ratio, density, shear wave velocity, shear module, and radius of foundation. The frequency independent expressions to determine the swaying and the rocking dashpots, and the corresponding springs of a rigid circular foundation are proposed, by [23]:

$c_{s}=\frac{4.6}{2-v_{s}} \rho_{s} V_{s} R_{F}^{2}$,

$c_{r}=\frac{0.4}{1-v_{s}} \rho_{s} V_{s} R_{F}^{4}$,

$k_{s}=\frac{8 G_{s} R_{F}}{2-v_{s}}$,

$k_{r}=\frac{8 G_{s} R_{F}^{3}}{3\left(1-v_{s}\right)}$,

where $c_{s}$ and $c_{r}$ represent dampings of the swaying and the rocking dashpots, and $k_{s}$ and $k_{r}$ denote the stiffnesses of the corresponding springs. The motion equation can be converted in state-space as [24]:

$\dot{Z}(t)=A Z(t)+B U(t)$

$Y(t)=R Z(t)+Q U(t)$

$Z(t)=\left\{\begin{array}{c}X(t) \\ \dot{X}(t)\end{array}\right\}$.

The $A$ and $B$ matrices are defined in the following equations. $R$ and $Q$ are dependent on the type of output $Y(t)$. The output vector can be considered displacement, velocity, and acceleration vector of the structural system. Transfer function matrix is obtained from the Laplace and Fourier transformation of the state-space is given in the frequency domain [24]:

$$
\begin{aligned}
& A=\left[\begin{array}{cc}
{[0]_{(N+3) *(N+3)}} & {[I]_{(N+3) *(N+3)}} \\
-M^{-1} K & -M^{-1} C
\end{array}\right], \\
& B=\left[\begin{array}{c}
{[0]_{(N+3) *(N+3)}} \\
-M^{-1} m^{*}
\end{array}\right], \\
& T F(s)=R(s I-A)^{-1} B+Q .
\end{aligned}
$$

\section{The optimization process}

The optimization procedure is used to obtain the optimum design of the TMD system. In this study, The Hळ norm of the acceleration transfer function is considered as the objective function. $\mathrm{H} \infty$ norm is defined in Eq. (17) for

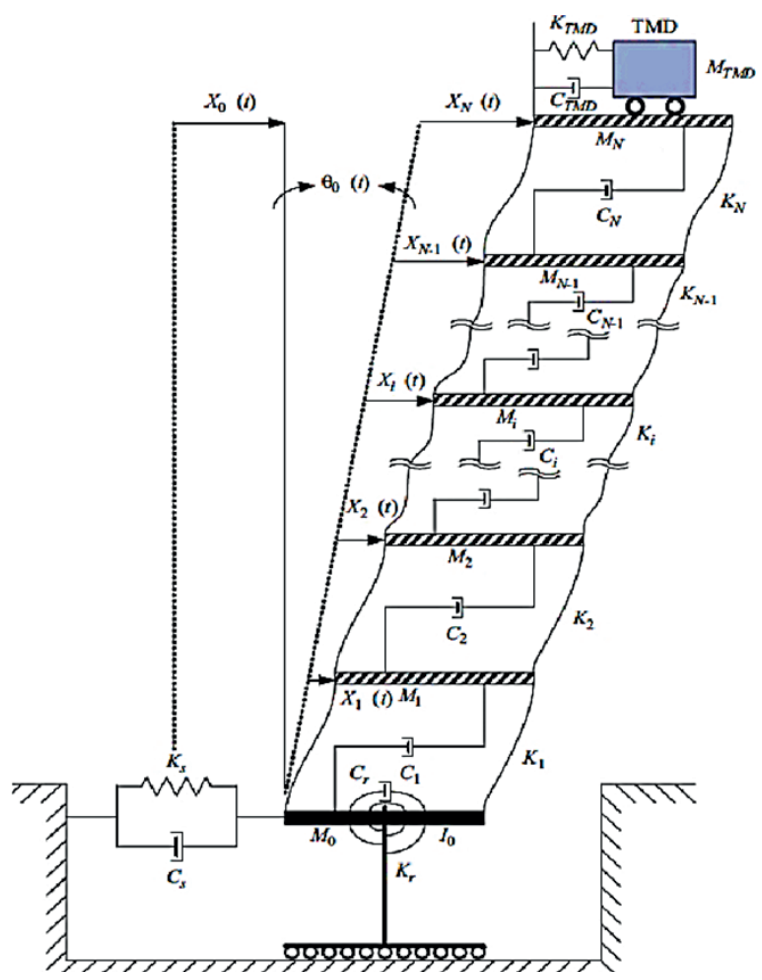

Fig. 1 Model of the SSI system with TMD

a multi-input multi-output (MIMO) system where $\bar{\sigma}$ is the largest singular value of the transfer function matrix and $\sup _{\omega}$ means the smallest higher bound of this relation over all frequencies [24]:

$T F_{\infty}=\sup _{\omega} \bar{\sigma}(T F(w))$.

For a structure with SSI effects, the responses of base and the effect of rotation must be added to find the total acceleration transfer function of the top story, so the objective function is considered as:

$f(x)=20 \log _{10} T F_{N}(w)+T F_{0}(w)+T F_{\theta}(w) z_{N \infty}$,

where $T F_{N}(\omega)$ is the acceleration transfer function of the Nth story. $T F_{0}(\omega)$ and $T F_{\theta}(\omega)$ are the acceleration transfer function of the base and rotation of the base, respectively. To find the optimum design of TMD, the period and damping ratio as the design variables are defined in Eqs. (23) and (24). The mass of TMD is considered to be a constant value during the optimization process. Due to the evaluation of the mass effect, the range of the mass of the TMD is taken between 1 and 4 percent of the total mass of the structure. The optimization algorithms are presented in the following.

$T_{d}=2 \pi \sqrt{\frac{m_{d}}{k_{d}}}$ 


$$
\xi_{d}=\frac{c_{d}}{2 m_{d} \sqrt{\frac{k_{d}}{m_{d}}}}
$$

\subsection{Colliding Bodies Optimization (CBO)}

This algorithm was firstly proposed by Kaveh and Mahdavi [25]. The CBO is a simple algorithm, and it does not require any internal parameters. This method is employed for the optimal design of truss structures with continuous and discrete variables [26]. The main idea of this algorithm is based on the momentum and energy conservation law for one-dimensional collision. One body with distinctive mass and velocity collides with the other one and they move towards a minimum energy level. The pseudo-code of the CBO optimizer is represented in the following steps [25]:

1. Initialize and create the population: The random positions of all $\mathrm{CBs}$ are generated in a search space.

2. Defining mass: For each $\mathrm{CB}$, the value of the body mass is defined.

3. Sort the bodies according to their mass;

4. Divide the bodies into stationary and moving groups: The velocity of stationary bodies before the collision is zero. Moving bodies move toward stationary ones and their velocities before collision are calculated.

5. The collision between bodies: The new velocities of stationary and moving bodies after the collision are calculated.

6. Updating CBs: The new position of each moving CB is calculated by the obtained velocity in the past step.

7. Check the stopping criterion: The optimization is repeated from Step 2 until the maximum iteration number is satisfied.

\subsection{Enhanced Colliding Bodies Optimization (ECBO)}

A modified version of the $\mathrm{CBO}$ was introduced by Kaveh and Ilchi Ghazaan [27]. ECBO has an additional memory to save the best solutions. Due to this memory, the speed of the convergence of ECBO increases with respect to standard $\mathrm{CBO}$ and makes the algorithm able to escape local optima. The high performance of this algorithm is presented in different applications [28]. The ECBO pseudo-code for a minimization problem is as follow [27]:

1. Initialization: The initial positions of all $\mathrm{CBs}$ are generated.

2. Mass values: The mass values of $\mathrm{CBs}$ are evaluated according to $\mathrm{CBO}$.
3. Saving: Some of the best CBs and their mass are saved in memory. Thus, the best solutions are saved in a Colliding Memory (CM). Then, the CM's solutions are added to the population, and the same numbers of the worst solution are removed. According to their masses, CBs of the population are sorted.

4. Creating groups: CBs are divided into two primary groups; i.e., stationary and moving groups.

5. Criteria before the collision: the velocities of the CBs before collision are obtained.

6. Criteria after the collision: The velocities of stationary and moving bodies after the collision are calculated.

The new location of each CB is evaluated.

7. Jump out of local optima: Meta-heuristic algorithms should have the ability to escape from the local optimum. A parameter called Pro is used in ECBO that is between 0 and 1 . It is specified whether a component of each CB must be changed or not.

8. Terminal condition check: The process of optimization after the predefined maximum number of evaluations is terminated. Otherwise, go to Step 2 for a new loop of iteration.

\subsection{Water Strider Algorithm (WSA)}

The WSA is a new and efficient meta-heuristic algorithm developed by Kaveh and Dadras Eslamlou [29]. The WSA is simulated in five steps by the water strider life including birth, territory establishment, mating, feeding mechanisms, and death. In this algorithm, a lake is a search-space and solutions are the territories of the lake. The optimization process was introduced for a maximization problem. It is noted that problems can easily be converted into minimization problems without changing in the optimum variables. The WSA pseudo-code for a maximization problem is as follow [29]:

1. Birth: The water striders (WSs) are randomly generated as agents in the lake. Then, the initial WSs are sorted by objective function as their fitness.

2. Territory establishment: WSs live in territories to mate and feed. The $n w s / n t$ groups are created by sorted WSs. The $j^{\text {th }}$ WS of each group lives in $j^{\text {th }}$ territory, where $j=1,2 \ldots, n t$. The number of the WSs and territories are taken as $n w s$ and $n t$; respectively. In each territory, the worst fitness is as the male (keystone) and the best positions are females because the females find the best place for feeding. 
3. Mating: The mating process is an important step in the water strider life. The probability of mating is assumed to equal $p=50 \%$. The new position of the keystone is updated.

4. Feeding: After the mating process, WSs wastes a lot of energy whether the mating takes place or not. In this step, the objective function based on the new position of the WS is evaluated to assess the food. If the value of the objective function is higher than the previous state, it means that it has found the food else it should move toward the best position.

5. Death and substitution: The result of the feeding process is evaluated and compared with the former position. If the new fitness is lesser, the keystone will die and the position of matured WS (as keystone) is initialized. Otherwise, the keystone would remain alive.

6. Termination: Finally, if the maximum evaluation number is satisfied, the process of optimization stops.

\subsection{Dynamic Water Strider Algorithm (DWSA)}

In the dynamic version of the water strider algorithm, the number of territories (nt) and approaching distances change during the optimization process to improve the behavior of the algorithm [30]. At the beginning of the search, the number of territories is considered a maximum number, because the WSs find the local optimum regions and improve their fitness. While in the last iterations, the number of territories tends toward a limited number of territories, but the total number of populations is fixed during the search process. Additionally, the keystone moves toward the best position, and the distance of them is decreased according to $(1-N A / \operatorname{Max} N A)^{2}$; where $N A$ and $\operatorname{Max} N A$ are the current and maximum number of analysis, respectively.

\subsection{Ray Optimization (RO) algorithm}

The Ray Optimization (RO) algorithm is developed as a population-based meta-heuristic conceptualized using the relationship between the angles of incidence and fraction based on Snell's law when light travels from a lighter medium to a darker medium [31]. In ray optimization, each agent is modeled as a ray of light that moves in the search space in order to find the global or near-global optimum solution. Also, Improved Ray Optimization (IRO) algorithm employs an approach for generating new solution vectors which has no limitation on the number of variables, so in the process of algorithm, there is no need to divide the variables into groups like RO [32]. In this study,
Ray Optimization (RO) algorithm is used because the problem has two design variables. The agents of RO are considered as beginning points of rays of light updated in the search space or traveled from a medium to another one based on Snell's light refraction law. Each ray of light is a vector so that its beginning point is the previous position of the agent in the search space, its direction and length is the searching step size in the current iteration, and its end point is the current position of the agent achieved by adding the step size to the beginning point. The new position of agents is updated to explore the search space and converge to the global or near-global optimum. In fact, RO aims to improve the quality of the solutions by refracting the rays toward the promising points obtained based on the best-known solution by each agent and all of them. The steps of Ray Optimization algorithm are as follows [31]:

1. Initialization: Randomly initialize the beginning point matrix of light rays $\left(L R^{t=0}\right)$ and their step size matrix.

2. Origin or Cockshy Point Making and Convergent Step: If the number of design variables $(n V)$ is more than 3 , the starting points $\left(L R_{i}\right)$ and step size vector of each agent should be divided into $\mathrm{k}$ subgroups. If $n V$ is an even number, the search space is divided into two-dimensional spaces, and if $n V$ is an odd number, the search space is divided into two-dimensional space(s) and one 3D space.

3. Stopping Criteria: If the stopping criterion of the algorithm is fulfilled, the search procedure terminates; otherwise, the algorithm returns to the second step and continues the search. The finishing criterion is considered here as the maximum number of objective function evaluations or the maximum number of algorithm iterations.

\subsection{Teaching-Learning-Based optimization (TLBO)}

The Teaching-learning-based optimization (TLBO) algorithm is based on the classical school learning process [33]. TLBO consists of two stages: the effect of a teacher on learners and the influence of learners on each other. In this algorithm, the initial population comprising of students or learners is selected randomly. In each iteration, the smartest student with the highest objective function is assigned as the teacher. Students are updated iteratively to search the optimum within two phases: based on the knowledge transfer from the teacher (teacher phase) and interaction with other students (learner phase). In TLBO the performance of the class in learning or the performance of the teacher in teaching is considered as a normal distribution 
of marks obtained by the students. TLBO improves other students in the teacher phase by employing the difference between the teacher's knowledge and the average knowledge of all the students. The knowledge of each student is obtained based on the position taken place by that student in the search space. In a class, students also improve themselves via interacting with each other after the teaching is completed. In the learner phase, the TLBO algorithm improves the quality of each student by the knowledge interaction between that student and another randomly selected one. The TLBO algorithm has only two parameters: number of learners $(n L)$ and maximum number of objective function evaluations. Since these two parameters exist in any other meta-heuristics, TLBO can be called a parameter-less meta-heuristic. These two phases are presented and formulated as follows [33]:

Generation or education of the new learners (newL) based on the teacher phase. The class performance as a normal distribution of grades obtained by students can be characterized with the mean value of the distribution. In this phase TLBO aims to improve the class performance by shifting the mean position of the class individuals toward the best learner which is considered as the teacher. This phase is the elitism or global search or intensification ability of the algorithm. In this regard, TLBO updates the learners by a step size toward the teacher obtained based on the difference between the teacher's position and the mean position of all students combining with randomization. Considering the mean position of students in the search space as MeanL.

Generating new learners (newL) or updating the knowledge of students by interacting with each other in the learner phase. In this phase, each student interacts with a randomly selected one $\left(L_{r p}\right)$ except him or her for possible improvement of knowledge. After comparison, the student will be moved toward the randomly selected one if it is smarter $\left(\right.$ PFit $_{i}<$ PFit $\left.t_{r p}\right)$ and shifted away otherwise. The learner phase is the diversification capability of the algorithm by which each individual tries to improve by searching its neighborhood and sharing information with one randomly selected individual. The step size of the search will be decreased gradually as the students approach each other with the progress of the algorithm.

\subsection{Plasma Generation Optimization (PGO) algorithm}

The plasma generation optimization (PGO) as a newly developed physics-based meta-heuristic algorithm is applied [34]. PGO is a population-based optimizer inspired by the process of plasma generation. In this optimization method, each agent is modeled as an electron. The movement of electrons and changing their energy level are performed based on simulating the process of excitation, de-excitation, and ionization. These processes occur iteratively through plasma generation. The main steps of the PGO algorithm can be stated as follows [34]:

1. Initialization: At the start of the algorithm, electron beams bombard the atoms and initialize a population of $\mathrm{n}$ random candidate solutions from electrons with the different energy levels in the search space.

2. Specifying the physical process for each electron: To calculate the new position of each electron, a random number is firstly generated between 0 and 1 (rand1). The randomly generated number specifies which physical process should occur for the electron (i.e., excitation/de-excitation processes or ionization process).

3. Generating a new position of the electron based on excitation and/or De-excitation processes: In excitation process, atoms are made up of two charged particles: positive charge (protons) and negative charge (electron). Based on the behavior of electron waves, there are electrons in atomic excitation process that indicates the intensification ability of the algorithm by decreasing the size of d-orbitals. In de-excitation process, due to electron beams interaction with gas atoms, percent of the excited electrons lost their energy by emitting light such that their positions are changed from high-energy levels to low-energy levels.

4. Generate a new position of the electron based on the ionization process: High-energy electron beams collide with atoms. Some of these atomic electrons are ripped from the atom to immerse in the plasma. Due to the high kinetic energy of immersed electrons, they collide with other atoms. Therefore, the corresponding atoms are excited and become ions. This process is modeled by electrons movement which obeys from levy flight. In other words, trajectories of the electrons immersed in plasma obey levy distribution so that their movement can be mathematically simulated.

5. Updating the electron's position: According to the previous step, the new position of the electron is calculated. After that, the newly generated electron is compared with the previous one in the previous iteration. If the newly generated one is better, it will be replaced. On the other hand, the best electron in each 
iteration is compared with the best electron obtained so far, and if the best electron is better than the best one obtained so far, it is replaced.

6. Checking termination condition: If the number of iterations becomes more than the maximum number of iterations as the stopping criterion, the optimization process will be terminated, and the best solution obtained so far will be reported. Otherwise, it returns to Step 2 for the next round of iteration.

\section{Numerical example}

In this section, the optimum TMD system for a 40 -story shear building structure is designed using CBO, ECBO, WAS, DWSA, RO, TLBO, and PGO. The properties of the structure system including mass, moment of inertia, height of the stories, and the parameters of the circular foundation of the structure including radius $\left(R_{F}\right)$, mass $\left(m_{0}\right)$ and mass moment of inertia $\left(I_{0}\right)$ are shown in Table 1 [35]. The stiffness and damping of the stories linearly decrease with increasing $z_{i}$. Three soil types under structure are considered and soil properties are presented in Tables 2 and 3, where $v_{s}$ is Poisson's ratio; Gs is shear modulus; $\rho_{s}$ is mass density; $V_{s}=G_{s} / \rho_{s}$ is the shear wave velocity [35].

To minimize the infinity norm of the acceleration transfer function of the top story, the period, and the damping ratio of the TMD system are considered as design variables.

Table 1 Properties of the 40-story shear building [35]

\begin{tabular}{lc}
\hline$z_{1}-z_{40}(m)$ & $4-160$ \\
$m_{i}(t)$ & 980 \\
$I_{i}\left(\mathrm{kgm}^{2}\right)$ & $1.31 \times 10^{8}$ \\
$k_{1}-k_{40}(M N / m)$ & $2130-998$ \\
$c_{1}-c_{40}(M N s / m)$ & $42.6-20$ \\
$R_{F}(m)$ & 20 \\
$m_{0}(t)$ & 1960 \\
$I_{0}\left(\mathrm{kgm}^{2}\right)$ & $1.96 \times 10^{8}$ \\
\hline
\end{tabular}

Table 2 Parameters of the soil types [35]

\begin{tabular}{lcccc}
\hline Soil type & $v_{s}$ & $G_{s}\left(\mathrm{~N} / \mathrm{m}^{2}\right)$ & $\rho_{s}\left(\mathrm{~kg} / \mathrm{m}^{3}\right)$ & $V_{s}(\mathrm{~m} / \mathrm{s})$ \\
\hline Soft soil & 0.49 & $1.80 \times 10^{7}$ & 1800 & 100 \\
Medium soil & 0.48 & $1.71 \times 10^{8}$ & 1900 & 300 \\
Dense soil & 0.33 & $6.00 \times 10^{8}$ & 2400 & 500 \\
\hline
\end{tabular}

Table 3 Stiffness and damping

\begin{tabular}{lcccc}
\hline Soil type & $c_{s}(\mathrm{Ns} / \mathrm{m})$ & $c_{r}(\mathrm{Ns} / \mathrm{m})$ & $k_{s}(\mathrm{~N} / \mathrm{m})$ & $k_{r}(\mathrm{~N} / \mathrm{m})$ \\
\hline Soft soil & $2.19 \times 10^{8}$ & $2.26 \times 10^{10}$ & $1.9 \times 10^{9}$ & $7.53 \times 10^{11}$ \\
Medium soil & $6.90 \times 10^{8}$ & $7.02 \times 10^{10}$ & $1.80 \times 10^{10}$ & $7.02 \times 10^{12}$ \\
Dense soil & $1.32 \times 10^{9}$ & $1.15 \times 10^{11}$ & $5.75 \times 10^{10}$ & $1.91 \times 10^{13}$ \\
\hline
\end{tabular}

The different TMDs are designed for this optimization problem and the ranges of mass values are set according to the previous sections. The period of TMD $\left(T_{d}\right)$ is searched between 0.8 and 1.2 times the fundamental period of the fixed base structure and the damping ratio of the TMD system $\left(\xi_{d}\right)$ is within $[0.01,0.3]$. The maximum number of iterations is defined as the stopping criteria of the algorithms, which is considered equal to 100 for all algorithms but optimum results are obtained in less than 100 . For a reliable assessment, the statistical results are based on 10 independent trials for each task, and the tuning parameters of each algorithm are considered according to the source papers. In this study, the internal tuning parameters of mentioned algorithms are presented in Table 4.

The optimum designs are obtained by these algorithms given in Tables $5-8$. The best $\left(F_{\min }\right)$, average $\left(F_{\text {ave }}\right)$, and standard deviation (std) of the 10 independent trials are presented in Tables 5-8. Almost all algorithms are successful in finding the best results. A careful examination of results reveals that PGO and TLBO have better performance in accuracy compared to other used algorithms. These algorithms also have better results in terms of the average and standard deviation values. According to these tables, the $\mathrm{CBO}, \mathrm{ECBO}$, and $\mathrm{RO}$ are worse than the others in robustness because of the average values and standard deviations. Additionally, the investigations show that the optimum parameters depend on the soil type, especially soft soil. It is noted that with the increase in softness of soil, the TMD effect is increased. The infinity norm of the

Table 4 Suitable values for the parameters of the algorithms

\begin{tabular}{|c|c|c|}
\hline Algorithm & Parameters & Values \\
\hline $\mathrm{CBO}$ & Pop. size & 20 \\
\hline \multirow{3}{*}{ ECBO } & Pop. size & 20 \\
\hline & CM size & 2 \\
\hline & Pro & 0.25 \\
\hline \multirow{3}{*}{ WSA } & $n w s$ & 20 \\
\hline & $n t$ & 10 \\
\hline & $p$ & 0.5 \\
\hline \multirow{3}{*}{ DWSA } & $n w s$ & 40 \\
\hline & $n t$ & 20 \\
\hline & $p$ & 0.5 \\
\hline RO & Pop. size & 20 \\
\hline TLBO & Pop. size & 20 \\
\hline \multirow{4}{*}{ PGO } & Pop. size & 20 \\
\hline & EDR & 0.6 \\
\hline & DR & 0.3 \\
\hline & DRS & 0.15 \\
\hline
\end{tabular}


Table 5 The results of the optimization process for $m_{d}=392(t)$

\begin{tabular}{|c|c|c|c|c|c|c|c|c|}
\hline Case & & $\mathrm{CBO}$ & ECBO & WSA & DWSA & $\mathrm{RO}$ & TLBO & PGO \\
\hline \multirow{6}{*}{ Fixed Based } & $m_{d}$ & 392 & 392 & 392 & 392 & 392 & 392 & 392 \\
\hline & $T_{d}(s)$ & 3.8794576 & 3.8794672 & 3.8794610 & 3.8794474 & 3.8794923 & 3.8794569 & 3.8794487 \\
\hline & $\xi_{d}$ & 0.0953674 & 0.0953995 & 0.0953780 & 0.0953322 & 0.0954873 & 0.0953653 & 0.0953359 \\
\hline & $F_{\min }$ & 19.8889 & 19.8889 & 19.8889 & 19.8889 & 19.8889 & 19.8889 & 19.8889 \\
\hline & $F_{\text {ave }}$ & 19.8890 & 19.8891 & 19.8889 & 19.8889 & 19.8890 & 19.8889 & 19.8889 \\
\hline & std & $1.141 \mathrm{E}-4$ & $1.870 \mathrm{E}-4$ & $6.171 \mathrm{E}-6$ & $7.390 \mathrm{E}-6$ & $8.4080 \mathrm{E}-5$ & $3.3164 \mathrm{E}-7$ & $1.2144 \mathrm{E}-6$ \\
\hline \multirow{6}{*}{ Dense Soil } & $m_{d}$ & 392 & 392 & 392 & 392 & 392 & 392 & 392 \\
\hline & $T_{d}(s)$ & 3.9767001 & 3.9764704 & 3.9766866 & 3.9766975 & 3.9766899 & 3.9766865 & 3.9766815 \\
\hline & $\xi_{d}$ & 0.0958235 & 0.0950418 & 0.0957796 & 0.0958152 & 0.0957904 & 0.0957794 & 0.0957629 \\
\hline & $F_{\text {min }}$ & 20.0000 & 20.0000 & 20.0000 & 20.0000 & 20.0000 & 20.0000 & 20.0000 \\
\hline & $F_{\text {ave }}$ & 20.0001 & 20.0003 & 20.0000 & 20.0001 & 20.0001 & 20.0000 & 20.0000 \\
\hline & std & $2.047 \mathrm{E}-4$ & $1.803 \mathrm{E}-4$ & $1.567 \mathrm{E}-6$ & $7.840 \mathrm{E}-4$ & $1.0518 \mathrm{E}-4$ & $2.5054 \mathrm{E}-6$ & $1.3189 \mathrm{E}-6$ \\
\hline \multirow{6}{*}{ Medium Soil } & $m_{d}$ & 392 & 392 & 392 & 392 & 392 & 392 & 392 \\
\hline & $T_{d}(s)$ & 4.1401948 & 4.1401842 & 4.1402482 & 4.1401937 & 4.1401928 & 4.1401912 & 4.1401917 \\
\hline & $\xi_{d}$ & 0.0965458 & 0.0965137 & 0.0967013 & 0.0965426 & 0.0965399 & 0.0965349 & 0.0965367 \\
\hline & $F_{\min }$ & 20.1193 & 20.1193 & 20.1193 & 20.1193 & 20.1193 & 20.1193 & 20.1193 \\
\hline & $F_{\text {ave }}$ & 20.1194 & 20.1194 & 20.1193 & 20.1193 & 20.1193 & 20.1193 & 20.1193 \\
\hline & std & $1.000 \mathrm{E}-4$ & $1.813 \mathrm{E}-4$ & $2.017 \mathrm{E}-6$ & $2.135 \mathrm{E}-6$ & $5.4116 \mathrm{E}-5$ & $1.0935 \mathrm{E}-6$ & $3.2104 \mathrm{E}-6$ \\
\hline \multirow{6}{*}{ Soft Soil } & $m_{d}$ & 392 & 392 & 392 & 392 & 392 & 392 & 392 \\
\hline & $T_{d}(s)$ & 5.8890745 & 5.8891531 & 5.8892250 & 5.8891018 & 5.8891880 & 5.8891125 & 5.8890884 \\
\hline & $\xi_{d}$ & 0.1013467 & 0.1014790 & 0.1015993 & 0.1013937 & 0.1015387 & 0.1014115 & 0.1013712 \\
\hline & $F_{\text {min }}$ & 20.1261 & 20.1261 & 20.1261 & 20.1261 & 20.1261 & 20.1261 & 20.1261 \\
\hline & $F_{\text {ave }}$ & 20.1262 & 20.1264 & 20.1261 & 20.1261 & 20.1262 & 10.1261 & 10.1261 \\
\hline & std & $1.821 \mathrm{E}-4$ & $4.514 \mathrm{E}-4$ & $1.003 \mathrm{E}-5$ & $2.806 \mathrm{E}-6$ & $7.6212 \mathrm{E}-5$ & $8.4505 \mathrm{E}-7$ & $2.9262 \mathrm{E}-6$ \\
\hline
\end{tabular}

Table 6 The results of the optimization process for $m_{d}=784(t)$

\begin{tabular}{|c|c|c|c|c|c|c|c|c|}
\hline Case & & $\mathrm{CBO}$ & ECBO & WSA & DWSA & $\mathrm{RO}$ & TLBO & PGO \\
\hline \multirow{6}{*}{ Fixed Based } & $m_{d}$ & 784 & 784 & 784 & 784 & 784 & 784 & 784 \\
\hline & $T_{d}(s)$ & 3.9303268 & 3.9302939 & 3.9303374 & 3.9303237 & 3.9303486 & 3.9303416 & 3.9303383 \\
\hline & $\xi_{d}$ & 0.1332760 & 0.1331761 & 0.1333058 & 0.1332675 & 0.1333373 & 0.1331774 & 0.1333086 \\
\hline & $F_{\text {min }}$ & 17.5094 & 17.5094 & 17.5094 & 17.5094 & 17.5094 & 17.5094 & 17.5094 \\
\hline & $F_{\text {ave }}$ & 17.5095 & 17.5095 & 17.5094 & 17.5094 & 17.5094 & 17.5094 & 17.5094 \\
\hline & $s t d$ & $1.664 \mathrm{E}-4$ & $1.377 \mathrm{E}-4$ & $6.320 \mathrm{E}-6$ & $6.264 \mathrm{E}-5$ & $6.9856 \mathrm{E}-5$ & $1.0993 \mathrm{E}-6$ & 2.3194E-6 \\
\hline \multirow{6}{*}{ Dense Soil } & $m_{d}$ & 784 & 784 & 784 & 784 & 784 & 784 & 784 \\
\hline & $T_{d}(s)$ & 4.0298350 & 4.0296530 & 4.0298913 & 4.0298726 & 4.0298369 & 4.0298656 & 4.0298772 \\
\hline & $\xi_{d}$ & 0.1338760 & 0.1333885 & 0.1340242 & 0.1339757 & 0.1338818 & 0.1339571 & 0.1339877 \\
\hline & $F_{\min }$ & 17.5888 & 17.5888 & 17.5888 & 17.5888 & 17.5888 & 17.5888 & 17.5888 \\
\hline & $F_{\text {ave }}$ & 17.5889 & 17.5889 & 17.5888 & 17.5888 & 17.5889 & 17.5888 & 17.5888 \\
\hline & std & $1.308 \mathrm{E}-4$ & $8.826 \mathrm{E}-5$ & $3.465 \mathrm{E}-6$ & $1.508 \mathrm{E}-4$ & $1.3643 \mathrm{E}-4$ & $2.0959 \mathrm{E}-6$ & $6.3070 \mathrm{E}-6$ \\
\hline \multirow{6}{*}{ Medium Soil } & $m_{d}$ & 784 & 784 & 784 & 784 & 784 & 784 & 784 \\
\hline & $T_{d}(s)$ & 4.1906860 & 4.1971115 & 4.1971550 & 4.1910422 & 4.1970222 & 4.1971071 & 4.1970857 \\
\hline & $\xi_{d}$ & 0.1348949 & 0.1349924 & 0.1351006 & 0.1349795 & 0.1347836 & 0.1349863 & 0.1349358 \\
\hline & $F_{\text {min }}$ & 17.6726 & 17.6726 & 17.6726 & 17.6726 & 17.6726 & 17.6726 & 17.6726 \\
\hline & $F_{\text {ave }}$ & 17.6731 & 17.6727 & 17.6726 & 17.6726 & 17.6727 & 17.6726 & 17.6726 \\
\hline & std & $1.157 \mathrm{E}-4$ & $1.130 \mathrm{E}-4$ & $2.127 \mathrm{E}-5$ & $5.430 \mathrm{E}-5$ & $6.8007 \mathrm{E}-5$ & $8.1359 \mathrm{E}-7$ & $3.8734 \mathrm{E}-6$ \\
\hline \multirow{6}{*}{ Soft Soil } & $m_{d}$ & 784 & 784 & 784 & 784 & 784 & 784 & 784 \\
\hline & $T_{d}(s)$ & 5.9828314 & 5.9827290 & 5.9827409 & 5.9830355 & 5.9828485 & 5.9829304 & 5.9829474 \\
\hline & $\xi_{d}$ & 0.1411784 & 0.1410380 & 0.1410545 & 0.1414524 & 0.1412015 & 0.1413117 & 0.1413343 \\
\hline & $F_{\min }$ & 17.6460 & 17.6460 & 17.6460 & 17.6460 & 17.6460 & 17.6460 & 17.6460 \\
\hline & $F_{\text {ave }}$ & 17.6467 & 17.6462 & 17.6460 & 17.6460 & 17.6461 & 17.6460 & 17.6460 \\
\hline & std & $9.073 \mathrm{E}-4$ & $2.257 \mathrm{E}-4$ & $9.198 \mathrm{E}-6$ & 5.374E-5 & $1.0568 \mathrm{E}-4$ & $1.3819 \mathrm{E}-6$ & 3.5373E-6 \\
\hline
\end{tabular}


Table 7 The results of the optimization process for $m_{d}=1176(t)$

\begin{tabular}{|c|c|c|c|c|c|c|c|c|}
\hline Case & & $\mathrm{CBO}$ & ECBO & WSA & DWSA & RO & TLBO & PGO \\
\hline \multirow{6}{*}{ Fixed Based } & $m_{d}$ & 1176 & 1176 & 1176 & 1176 & 1176 & 1176 & 1176 \\
\hline & $T_{d}(s)$ & 3.9815712 & 3.9816681 & 3.9816195 & 3.9816123 & 3.9816301 & 3.9815986 & 3.9816215 \\
\hline & $\xi_{d}$ & 0.1621598 & 0.1623825 & 0.1622748 & 0.1622579 & 0.1623002 & 0.1622253 & 0.1622796 \\
\hline & $F_{\min }$ & 16.0702 & 16.0702 & 16.0702 & 16.0702 & 16.0702 & 16.0702 & 16.0702 \\
\hline & $F_{\text {ave }}$ & 16.0703 & 16.0704 & 16.0702 & 16.0702 & 16.0703 & 16.0701 & 16.0702 \\
\hline & std & $1.148 \mathrm{E}-4$ & $1.159 \mathrm{E}-4$ & 4.199E-6 & $2.461 \mathrm{E}-5$ & $5.1932 \mathrm{E}-5$ & $2.2955 \mathrm{E}-6$ & $9.5751 \mathrm{E}-6$ \\
\hline \multirow{6}{*}{ Dense Soil } & $m_{d}$ & 1176 & 1176 & 1176 & 1176 & 1176 & 1176 & 1176 \\
\hline & $T_{d}(s)$ & 4.0833867 & 4.0833257 & 4.0834965 & 4.0834394 & 4.0834542 & 4.0833866 & 4.0834079 \\
\hline & $\xi_{d}$ & 0.1629682 & 0.1628298 & 0.1632114 & 0.1630857 & 0.1631185 & 0.1629678 & 0.1630150 \\
\hline & $F_{\min }$ & 16.1332 & 16.1333 & 16.1332 & 16.1332 & 16.1333 & 16.1333 & 16.1333 \\
\hline & $F_{\text {ave }}$ & 16.3334 & 16.1335 & 16.1332 & 16.1333 & 16.1334 & 16.1333 & 16.1333 \\
\hline & std & $1.154 \mathrm{E}-4$ & $2.264 \mathrm{E}-4$ & $1.735 \mathrm{E}-6$ & $5.071 \mathrm{E}-5$ & $1.2414 \mathrm{E}-4$ & $2.5960 \mathrm{E}-6$ & $1.2175 \mathrm{E}-5$ \\
\hline \multirow{6}{*}{ Medium Soil } & $m_{d}$ & 1176 & 1176 & 1176 & 1176 & 1176 & 1176 & 1176 \\
\hline & $T_{d}(s)$ & 4.2543038 & 4.2542581 & 4.2543533 & 4.2543479 & 4.2543554 & 4.2543519 & 4.2543527 \\
\hline & $\xi_{d}$ & 0.1640888 & 0.1639887 & 0.1641889 & 0.1641783 & 0.1641938 & 0.1641865 & 0.1641881 \\
\hline & $F_{\min }$ & 16.1985 & 16.1985 & 16.1985 & 16.1985 & 16.1985 & 16.1985 & 16.1985 \\
\hline & $F_{\text {ave }}$ & 16.1987 & 16.1987 & 16.1985 & 16.1985 & 16.1985 & 16.1985 & 16.1985 \\
\hline & std & $3.393 \mathrm{E}-4$ & $3.987 \mathrm{E}-4$ & $5.446 \mathrm{E}-6$ & $5.1960 \mathrm{E}-5$ & $3.1001 \mathrm{E}-5$ & $2.6910 \mathrm{E}-6$ & $4.2520 \mathrm{E}-6$ \\
\hline \multirow{6}{*}{ Soft Soil } & $m_{d}$ & 1176 & 1176 & 1176 & 1176 & 1176 & 1176 & 1176 \\
\hline & $T_{d}(s)$ & 6.0769885 & 6.0767115 & 6.0767396 & 6.0767786 & 6.0765129 & 6.0768141 & 6.0767729 \\
\hline & $\xi_{d}$ & 0.1715481 & 0.1712275 & 0.1712644 & 0.1713093 & 0.1710031 & 0.1713492 & 0.1713027 \\
\hline & $F_{\min }$ & 16.1475 & 16.1475 & 16.1475 & 16.1475 & 16.1475 & 16.1475 & 16.1475 \\
\hline & $F_{\text {ave }}$ & 16.1478 & 16.1478 & 16.1475 & 16.1475 & 16.1476 & 16.1475 & 16.1475 \\
\hline & std & $3.715 \mathrm{E}-4$ & $5.243 \mathrm{E}-4$ & $1.933 \mathrm{E}-5$ & $5.664 \mathrm{E}-5$ & $7.2158 \mathrm{E}-5$ & $2.8933 \mathrm{E}-6$ & $3.5373 \mathrm{E}-6$ \\
\hline
\end{tabular}

Table 8 The results of the optimization process for $m_{d}=1568(t)$

\begin{tabular}{|c|c|c|c|c|c|c|c|c|}
\hline Case & & $\mathrm{CBO}$ & ECBO & WSA & DWSA & RO & TLBO & PGO \\
\hline \multirow{6}{*}{ Fixed Based } & $m_{d}$ & 1568 & 1568 & 1568 & 1568 & 1568 & 1568 & 1568 \\
\hline & $T_{d}(s)$ & 4.0334551 & 4.0327852 & 4.0332090 & 4.0332799 & 4.0332530 & 4.0332951 & 4.0332772 \\
\hline & $\xi_{d}$ & 0.1868482 & 0.1854429 & 0.1863410 & 0.1864895 & 0.1864339 & 0.1865209 & 0.1864834 \\
\hline & $F_{\min }$ & 15.0368 & 15.0368 & 15.0368 & 15.0368 & 15.0368 & 15.0368 & 15.0368 \\
\hline & $F_{\text {ave }}$ & 15.0372 & 15.0368 & 15.0368 & 15.0368 & 15.0369 & 15.0368 & 15.0368 \\
\hline & std & $7.684 \mathrm{E}-4$ & $1.359 \mathrm{E}-4$ & $2.309 \mathrm{E}-5$ & $2.636 \mathrm{E}-5$ & $6.2963 \mathrm{E}-5$ & $1.3185 \mathrm{E}-6$ & $5.6475 \mathrm{E}-6$ \\
\hline \multirow{6}{*}{ Dense Soil } & $m_{d}$ & 1568 & 1568 & 1568 & 1568 & 1568 & 1568 & 1568 \\
\hline & $T_{d}(s)$ & 4.1373407 & 4.1372894 & 4.1373072 & 4.1373066 & 4.1373487 & 4.1373069 & 4.1373004 \\
\hline & $\xi_{d}$ & 0.1873448 & 0.1872422 & 0.1872797 & 0.1872788 & 0.1873605 & 0.1872792 & 0.1872663 \\
\hline & $F_{\min }$ & 15.0889 & 15.0889 & 15.0889 & 15.0889 & 15.0889 & 15.0889 & 15.0889 \\
\hline & $F_{\text {ave }}$ & 15.0894 & 15.0891 & 15.0889 & 15.0890 & 15.0890 & 15.0889 & 15.0890 \\
\hline & std & $8.304 \mathrm{E}-4$ & $1.240 \mathrm{E}-4$ & $1.7436 \mathrm{E}-5$ & $1.058 \mathrm{E}-4$ & $1.1215 \mathrm{E}-4$ & $5.9555 \mathrm{E}-7$ & $2.7319 \mathrm{E}-6$ \\
\hline \multirow{6}{*}{ Medium Soil } & $m_{d}$ & 1568 & 1568 & 1568 & 1568 & 1568 & 1568 & 1568 \\
\hline & $T_{d}(s)$ & 4.3119056 & 4.3119251 & 4.3120721 & 4.3119055 & 4.3119403 & 4.3119144 & 4.3119245 \\
\hline & $\xi_{d}$ & 0.1885080 & 0.1885425 & 0.1888004 & 0.1885079 & 0.1885698 & 0.1885237 & 0.1885413 \\
\hline & $F_{\min }$ & 15.1415 & 15.1415 & 15.1415 & 15.1415 & 15.1415 & 15.1415 & 15.1415 \\
\hline & $F_{\text {ave }}$ & 15.1421 & 15.1419 & 15.1415 & 15.1417 & 15.1417 & 15.1415 & 15.1415 \\
\hline & std & $8.640 \mathrm{E}-4$ & $5.157 \mathrm{E}-4$ & $8.578 \mathrm{E}-6$ & $4.659 \mathrm{E}-4$ & $1.5445 \mathrm{E}-4$ & $1.7693 \mathrm{E}-5$ & $9.9105 \mathrm{E}-6$ \\
\hline \multirow{6}{*}{ Soft Soil } & $m_{d}$ & 1568 & 1568 & 1568 & 1568 & 1568 & 1568 & 1568 \\
\hline & $T_{d}(s)$ & 6.1706643 & 6.1707112 & 6.1707510 & 6.1706521 & 6.1706372 & 6.1704425 & 6.1705683 \\
\hline & $\xi_{d}$ & 0.1961534 & 0.1961845 & 0.1962411 & 0.1961413 & 0.1961262 & 0.1959285 & 0.1960563 \\
\hline & $F_{\min }$ & 15.0700 & 15.0700 & 15.0700 & 15.0700 & 15.0700 & 15.0700 & 15.0700 \\
\hline & $F_{\text {ave }}$ & 15.0705 & 15.0702 & 15.0700 & 15.0701 & 15.0702 & 15.0700 & 15.0700 \\
\hline & std & $8.787 \mathrm{E}-4$ & $3.922 \mathrm{E}-4$ & $7.266 \mathrm{E}-6$ & $1.754 \mathrm{E}-4$ & $2.1680 \mathrm{E}-4$ & $1.8868 \mathrm{E}-6$ & $1.6343 \mathrm{E}-6$ \\
\hline
\end{tabular}


transfer function of the top story structure with respect to the periods and damping ratios are calculated and shown in Figs. 2 to 5. The results have been normalized to the

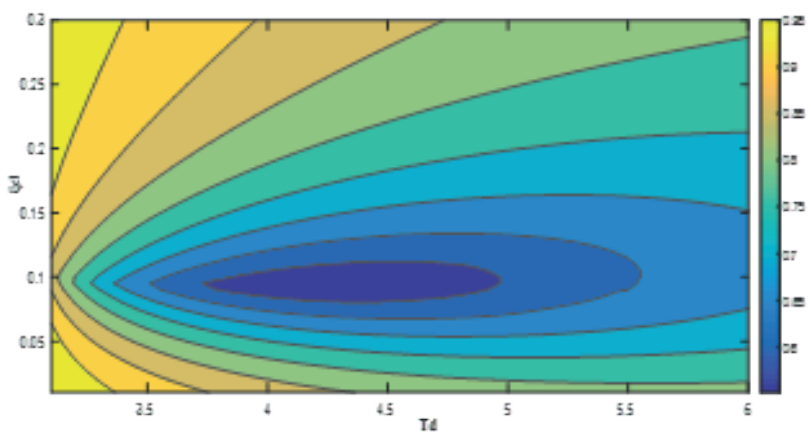

Fig. 2 Normalized infinity norm of the transfer function of the top story at $m_{d}=784(t)$ with respect to the design variables for fixed base structure

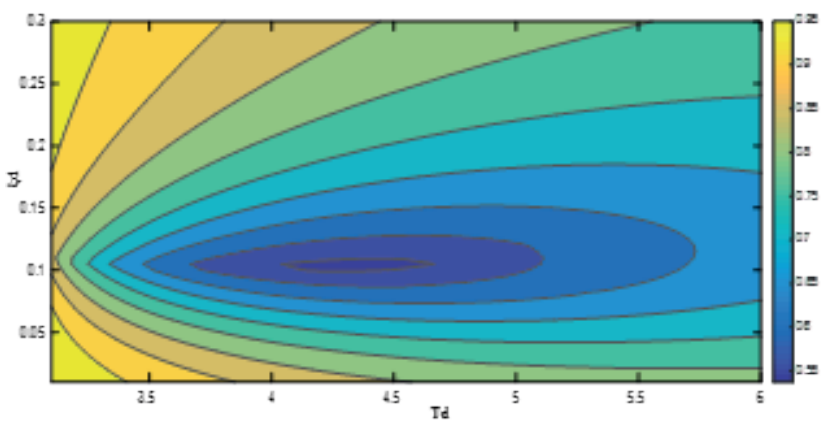

Fig. 3 Normalized infinity norm of the transfer function of the top story at $m_{d}=784(t)$ with respect to the design variables for dense soil type

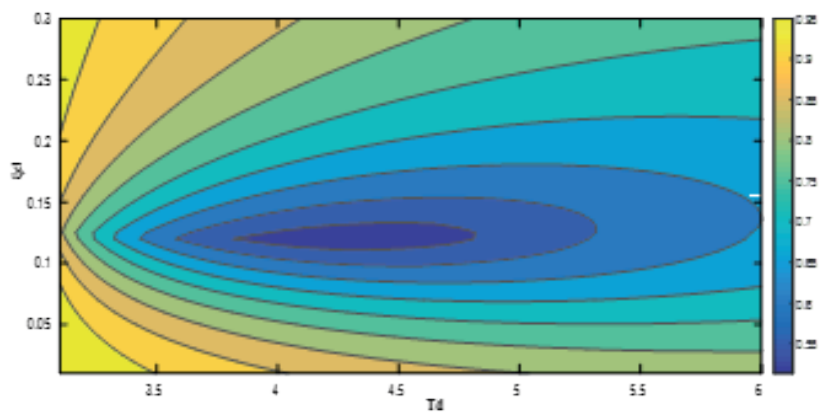

Fig. 4 Normalized infinity norm of the transfer function of the top story at $m_{d}=784(t)$ with respect to the design variables for medium soil

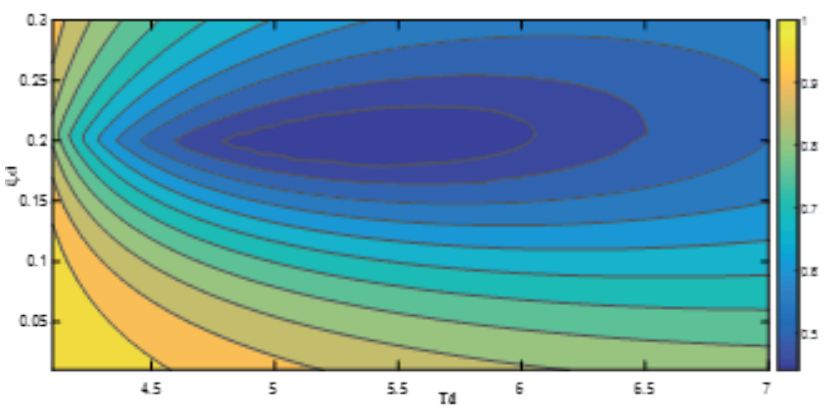

Fig. 5 Normalized infinity norm of the transfer function of the top story at $m_{d}=784(t)$ with respect to the design variables for soft soil type response of the uncontrolled structure and presented for different soil types. These figures show the optimum value in the search space. The optimization results show that the algorithms have close performances and all of them converge to solutions very close to the global optimum, which demonstrates the high performance of these algorithms.

Speed of convergence is an essential feature of algorithms. For this reason, the convergence history of the algorithms is displayed in Fig. 6. In this problem, the convergence speed of the optimization process is appropriate. As shown, these algorithms have a quick convergence, and it is evident that TLBO has the highest convergence rate in comparison with the other considered meta-heuristics because of a less number of analyses among algorithms. As shown, the convergence rates of the ECBO, WSA, DWSA, and PGO are higher than the $\mathrm{CBO}$ and RO.

\section{Performance of the optimum design in the time and frequency domain}

In the time domain, the main performance of the optimum design is evaluated by the top story displacement. The optimum result of the structure is tested by El-Centro record which is shown in Fig. 7. The maximum roof displacement of the controlled and uncontrolled structure under the El-Centro earthquake is represented in Table 9. It should be mentioned that the maximum roof displacement is considered relative displacement of the top story plus the displacement and rotation of the foundation.

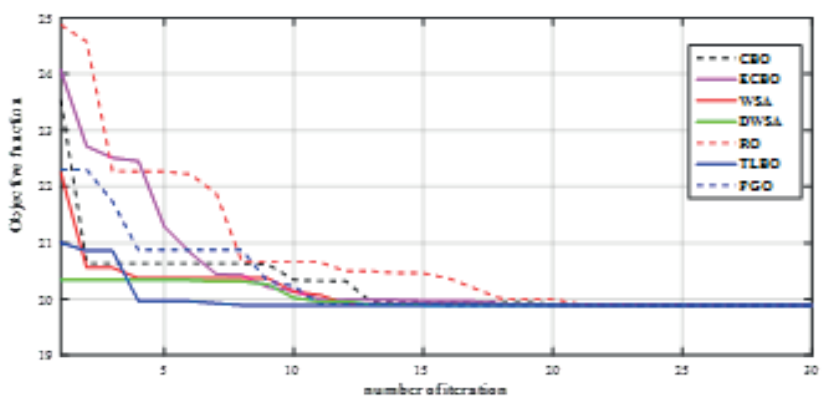

Fig. 6 Convergence history of the optimization methods for the fixed base structure and $m_{d}=392$

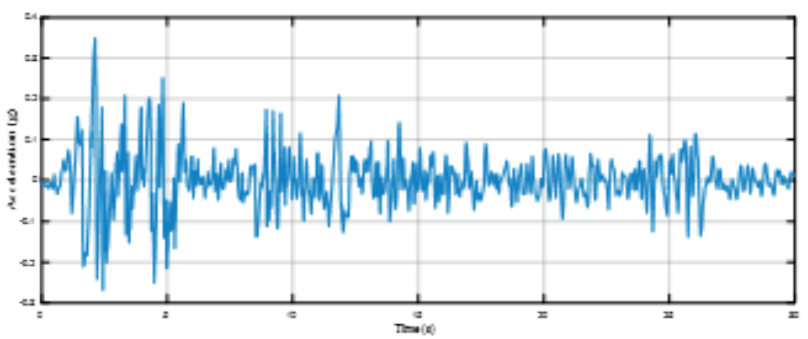

Fig. 7 Time history of El-Centro earthquake record (1940) 
According to Table 9, the reductions of maximum roof displacement are different values between $2.71 \%$ and $50.27 \%$. With increasing the mass of the TMD system, the maximum roof displacement reduces but a significant reduction is obtained for soft soil.

The main purpose of structural control is to reduce vibrations and the displacement stories when an earthquake occurs. To make a better comparison, the performance and efficiency of the TMDs, the time history, and the acceleration transfer function plots are shown in Figs. 8-15 for all support conditions. It is observed that the peak gain values of the transfer function are significantly reduced. As well as, the optimum results are tested by a historical earthquake. According to the figures, the displacement of the top story decreases with the increasing mass of the TMD. In general, the results indicate that the frequency domain base method is effective to decrease the responses in both frequency and time domains.

\section{Conclusions}

In this paper, seven population-based meta-heuristic algorithms are employed for the optimum design of the TMD system. The algorithms consist of colliding bodies optimization (CBO), enhanced colliding bodies optimization (ECBO), water strider algorithm (WSA), dynamic water strider algorithm (DWSA), ray optimization (RO), teaching-learning-based optimization (TLBO), plasma

Table 9 Maximum displacement of the top story under El-Centro (1940) earthquake

\begin{tabular}{lcccc}
\hline Case & $m_{d}(t)$ & $\begin{array}{c}\text { Roof } \\
\text { displacement } \\
\text { without TMD }\end{array}$ & $\begin{array}{c}\text { Roof } \\
\text { displacement } \\
\text { with TMD }\end{array}$ & $\begin{array}{c}\text { Percentage } \\
\text { of reduction }\end{array}$ \\
\hline Fixed & 782 & 0.2896 & 0.2817 & 2.73 \\
based & 1176 & 0.2896 & 0.2745 & 5.21 \\
& 1568 & 0.2896 & 0.2617 & 7.49 \\
& 392 & 0.2881 & 0.2803 & 9.63 \\
Dense soil & 784 & 0.2881 & 0.2731 & 5.21 \\
& 1176 & 0.2881 & 0.2664 & 7.53 \\
& 1568 & 0.2881 & 0.2601 & 9.72 \\
Medium & 784 & 0.3115 & 0.2756 & 11.52 \\
soil & 1176 & 0.3115 & 0.2619 & 15.92 \\
& 1568 & 0.3115 & 0.2556 & 17.94 \\
& 392 & 0.4776 & 0.3586 & 24.92 \\
& 784 & 0.4776 & 0.3022 & 36.73 \\
& 1176 & 0.4776 & 0.2630 & 44.93 \\
& 1568 & 0.4776 & 0.2375 & 50.27 \\
\hline
\end{tabular}

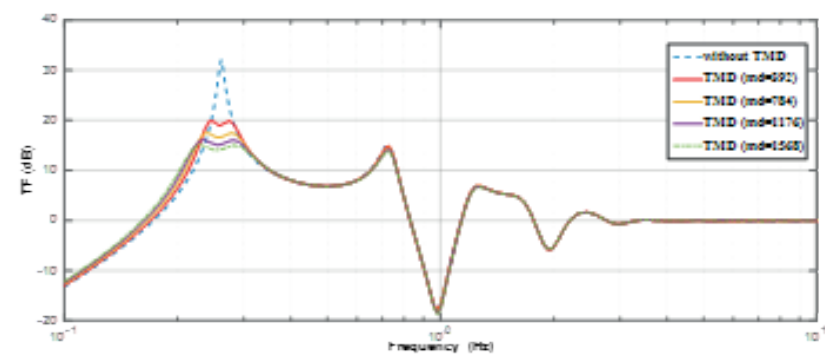

Fig. 8 Transfer function (TF) of the top story acceleration of the fixed based structure

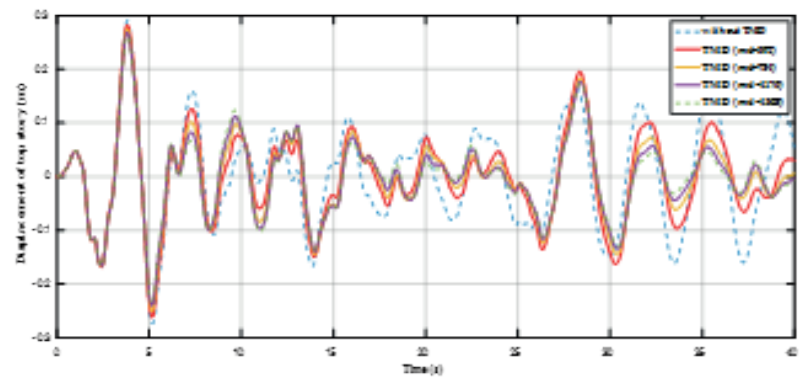

Fig. 9 Time history displacement of the top story of the fixed based structure

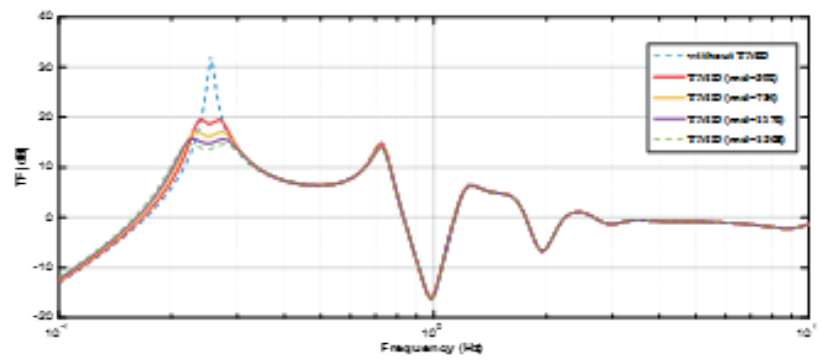

Fig. 10 Transfer function (TF) of the top story acceleration for dense soil

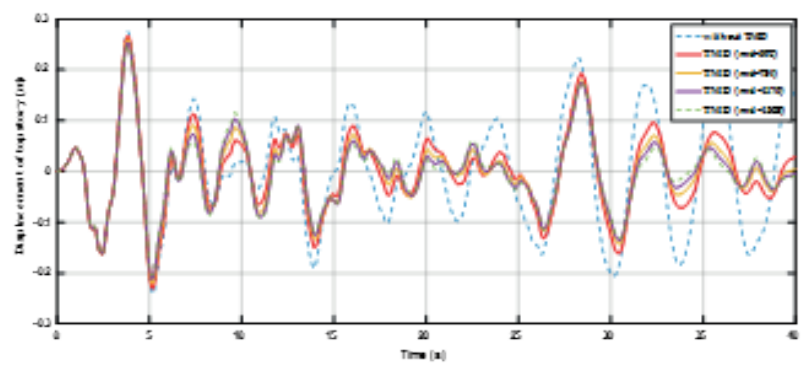

Fig. 11 Time history displacement of the top story for dense soil

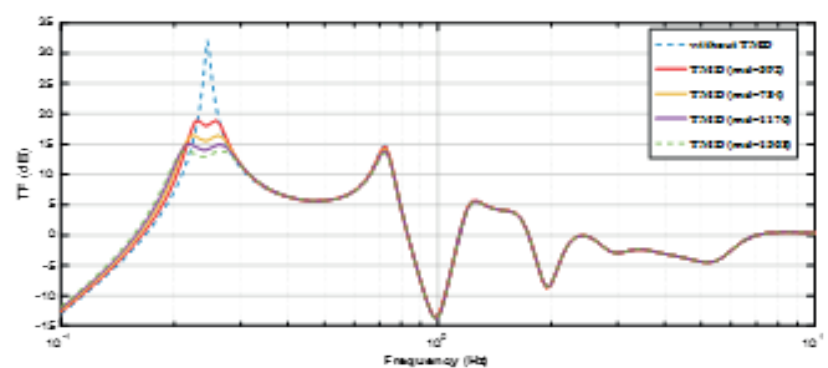

Fig. 12 Transfer function (TF) of the top story acceleration for medium soil 


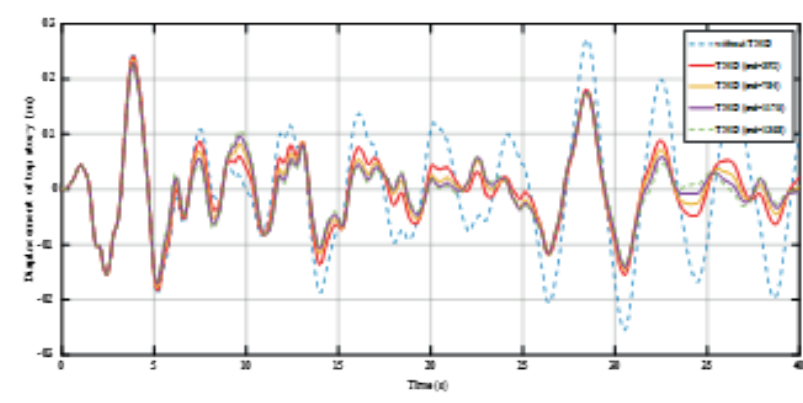

Fig. 13 Time history displacement of the top story for medium soil

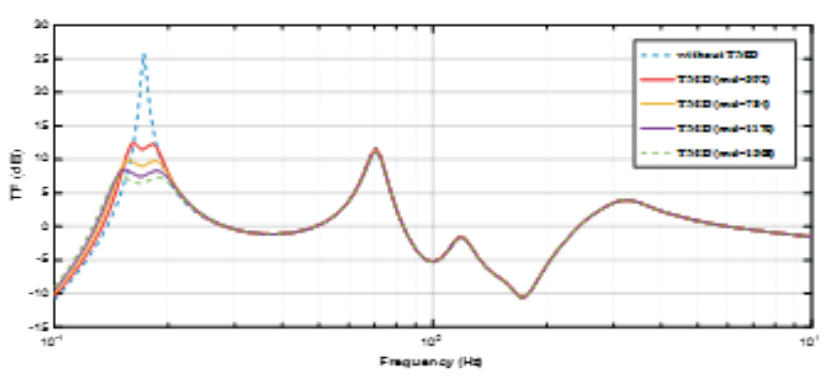

Fig. 14 Transfer function (TF) of the top story acceleration for soft soil

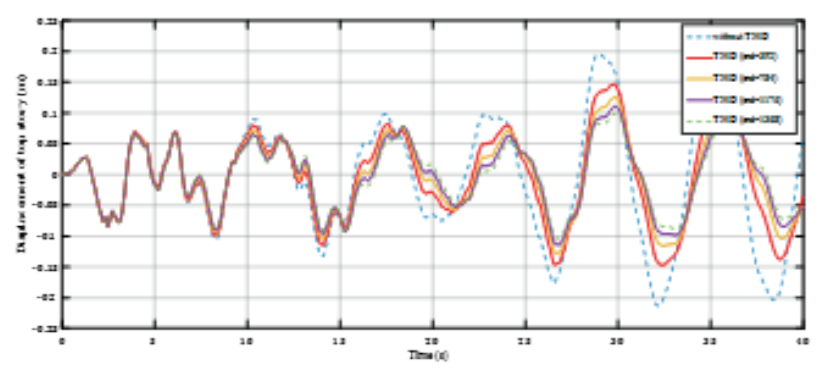

Fig. 15 Time history displacement of the top story for soft soil

\section{References}

[1] Talbi, E.-G. "Metaheuristics: From Design to Implementation", John Wiley \& Sons, Hoboken, NJ, USA, 2009.

[2] Kaveh, A. "Advances in Metaheuristic Algorithms for Optimal Design of Structures", Springer, Cham, Switzerland, 2014. https://doi.org/10.1007/978-3-319-05549-7

[3] Hadi, M. N. S., Arfiadi, Y. "Optimum Design of Absorber for MDOF Structures", Journal of Structural Engineering, 124(11), pp. 12721280, 1998.

https://doi.org/10.1061/(ASCE)0733-9445(1998)124:11(1272)

[4] Leung, A. Y. T., Zhang, H. "Particle swarm optimization of tuned mass dampers", Engineering Structures, 31(3), pp. 715-728, 2009. https://doi.org/10.1016/j.engstruct.2008.11.017

[5] Steinbuch, R. "Bionic optimisation of the earthquake resistance of high buildings by tuned mass dampers", Journal of Bionic Engineering, 8(3), pp. 335-344, 2011. https://doi.org/10.1016/S1672-6529(11)60036-X

[6] Bekdaş, G., Nigdeli, S. M. "Estimating optimum parameters of tuned mass dampers using harmony search", Engineering Structures, 33(9), pp. 2716-2723, 2011.

https://doi.org/10.1016/j.engstruct.2011.05.024 generation optimization (PGO) Algorithms. The objective of the optimization is to minimize the infinity norm of the transfer function of the structure. According to the results, almost all algorithms are successful in finding the best results. But, TLBO and PGO algorithms are more reliable and efficient than the others because they have the lowest average values and the lowest standard deviations in all cases of the current problem. However, the differences in average values with the other algorithms are almost $0.001 \mathrm{~dB}$. Furthermore, the convergence rate of all the utilized algorithms is appropriate, but TLBO presents the best results with a less number of analyses among the algorithms. The optimum free vibration parameters (period and damping ratio) are computed for different values of the TMD mass and different support conditions. The investigations show that the optimum parameters depend on the soil type, especially soft soil. The obtained values are evaluated in both frequency and time domains. The main performance of the optimum design is evaluated by the acceleration transfer function and the time history displacement of the top story. As shown, the mentioned algorithms have a good performance and effectiveness in the design of the TMD system.

\section{Compliance with ethical standards}

Conflict of interest: No potential conflict of interest was reported by the authors.

[7] Farshidianfar, A., Soheili, S. "Ant colony optimization of tuned mass dampers for earthquake oscillations of high-rise structures including soil-structure interaction", Soil Dynamics and Earthquake Engineering, 51, pp. 14-22, 2013. https://doi.org/10.1016/j.soildyn.2013.04.002

[8] Kaveh, A., Mohammadi, S., Hosseini, O. K., Keyhani, A., Kalatjari, V. R. "Optimum parameters of tuned mass dampers for seismic applications using charged system search", Iranian Journal of Science and Technology, Transactions of Civil Engineering, 39(C1), pp. $21-40,2015$. https://doi.org/10.22099/IJSTC.2015.2739

[9] Fahimi Farzam, M., Kaveh, A. "Optimum Design of Tuned Mass Dampers Using Colliding Bodies Optimization in Frequency Domain", Iranian Journal of Science and Technology, Transactions of Civil Engineering, 44(3), pp. 787-802, 2020. https://doi.org/10.1007/s40996-019-00296-6

[10] Anand, V., Satish Kumar, S. R. "Seismic Soil-structure Interaction: A State-of-the-Art Review", Structures, 16, pp. 317-326, 2018. https://doi.org/10.1016/j.istruc.2018.10.009 
[11] Farshidianfar, A. Soheili, S. "ABC optimization of TMD parameters for tall buildings with soil-structure interaction", Interaction and Multiscale Mechanics, 6(4), pp. 339-356, 2013.

https://oi.org/10.12989/imm.2013.6.4.339

[12] Farshidianfar, A. Soheili, S. "Optimization of TMD parameters for earthquake vibrations of tall buildings including soil-structure interaction", International Journal of Optimization in Civil Engineering, 3(3), pp. 409-429, 2013. [online] Available at: http://ijoce.iust.ac.ir/ article-1-141-fa.html

[13] Bekdaş, G. Nigdeli, S. M. "Metaheuristic based optimization of tuned mass dampers under earthquake excitation by considering soil-structure interaction", Soil Dynamics and Earthquake Engineering, 92, pp. 443-461, 2017. https://doi.org/10.1016/j.soildyn.2016.10.019

[14] Salvi, J., Pioldi, F., Rizzi, E. "Optimum Tuned Mass Dampers under seismic Soil-Structure Interaction", Soil Dynamics and Earthquake Engineering, 114, pp. 576-597, 2018. https://doi.org/10.1016/j.soildyn.2018.07.014

[15] Etedali, S., Akbari, M., Seifi, M. "MOCS-based optimum design of TMD and FTMD for tall buildings under near-field earthquakes including SSI effects", Soil Dynamics and Earthquake Engineering, 119, pp. 36-50, 2019.

https://doi.org/10.1016/j.soildyn.2018.12.027

[16] Li, C., Liu, Y. "Ground motion dominant frequency effect on the design of multiple tuned mass dampers", Journal of Earthquake Engineering, 8(1), pp. 89-105, 2004. https://doi.org/10.1080/13632460409350482

[17] Wang, J.-F., Lin, C.-C. "Seismic performance of multiple tuned mass dampers for soil-irregular building interaction systems", International Journal of Solids and Structures, 42(20), pp. 55365554,2005

https://doi.org/10.1016/j.ijsolstr.2005.02.042

[18] Li, C., Han, B. "Effect of dominant ground frequency and soil on multiple tuned mass dampers", The Structural Design of Tall and Special Buildings, 20(2), pp. 151-163, 2011. https://doi.org/10.1002/tal.519

[19] Kaveh, A., Fahimi Farzam, M., Jalali, H. H., Maroofiazar, R. "Robust optimum design of a tuned mass damper inerter", Acta Mechanica, 231(9), pp. 3871-3896, 2020. https://doi.org/10.1007/s00707-020-02720-9

[20] Kaveh, A., Fahimi Farzam, M., Jalali, H. H. "Statistical seismic performance assessment of tuned mass damper inerter", Structural Control and Health Monitoring, 27(10), Article ID e2602, 2020. https://doi.org/10.1002/stc.2602

[21] Kaveh, A., Fahimi Farzam, M., Maroofiazar, R. "Comparing H2 and $\mathrm{H} \infty$ Algorithms for Optimum Design of Tuned Mass Dampers under Near-Fault and Far-Fault Earthquake Motions", Periodica Polytechnica Civil Engineering, 64(3), pp. 828-844, 2020. https://doi.org/10.3311/PPci.16389

[22] Chopra, A. K. "Dynamics of Structures: Theory and Applications to Earthquake Engineering", Prentice-Hall, Upper Saddle River, NJ, USA, 1995.
[23] Wolf, J. P. "Foundation Vibration Analysis Using Simple Physical Models", Prentice-Hall, Englewood Cliffs, NJ, USA, 1994.

[24] Debnath, N., Deb, S. K., Dutta, A. "Frequency band-wise passive control of linear time invariant structural systems with $\mathrm{H} \infty$ optimization", Journal of Sound and Vibration, 332(23), pp. 6044-6062, 2013.

https://doi.org/10.1016/j.jsv.2013.06.018

[25] Kaveh, A., Mahdavi, V. R. "Colliding bodies optimization: A novel meta-heuristic method", Computers \& Structures, 139, pp. 18-27, 2014.

https://doi.org/10.1016/j.compstruc.2014.04.005

[26] Kaveh, A., Mahdavi, V. R. "Colliding Bodies Optimization: Extensions and Applications", Springer, Cham, Switzerland, 2015. https://doi.org/10.1007/978-3-319-19659-6

[27] Kaveh, A., Ilchi Ghazaan, M. "Enhanced colliding bodies optimization for design problems with continuous and discrete variables", Advances in Engineering Software, 77, pp. 66-75, 2014. https://doi.org/10.1016/j.advengsoft.2014.08.003

[28] Kaveh, A. "Applications of Metaheuristic Optimization Algorithms in Civil Engineering", Springer, Cham, Switzerland, 2017. https://doi.org/10.1007/978-3-319-48012-1

[29] Kaveh, A., Dadras Eslamlou, A. "Water strider algorithm: A new metaheuristic and applications", Structures, 25, pp. 520-541, 2020. https://doi.org/10.1016/j.istruc.2020.03.033

[30] Kaveh, A., Dadras Eslamlou, A., Khodadadi, N. "Dynamic Water Strider Algorithm for Optimal Design of Skeletal Structures", Periodica Polytechnica Civil Engineering, 64(3), pp. 904-916, 2020. https://doi.org/10.3311/PPci.16401

[31] Kaveh, A., Khayatazad, M. "A new meta-heuristic method: Ray Optimization", Computers \& Structures, 112-113, pp. 283-294, 2012. https://doi.org/10.1016/j.compstruc.2012.09.003

[32] Kaveh, A., Ilchi Ghazaan, M., Bakhshpoori, T. "An improved ray optimization algorithm for design of truss structures", Periodica Polytechnica Civil Engineering, 57(2), pp. 97-112, 2013. https://doi.org/10.3311/PPci.7166

[33] Rao, R. V., Savsani, V. J., Vakharia, D. P. "Teaching-learning-based optimization: A novel method for constrained mechanical design optimization problems", Computer-Aided Design, 43(3), pp. 303315, 2011. https://doi.org/10.1016/j.cad.2010.12.015

[34] Kaveh, A., Akbari, H., Hosseini, S. M. "Plasma generation optimization: a new physically-based metaheuristic algorithm for solving constrained optimization problems", Engineering Computations, 2020. https://doi.org/10.1108/EC-05-2020-0235

[35] Liu, M.-Y., Chiang, W.-L., Hwang, J.-H., Chu, C.-R. "Wind-induced vibration of high-rise building with tuned mass damper including soil-structure interaction", Journal of Wind Engineering and Industrial Aerodynamics, 96(6-7), pp. 1092-1102, 2008. https://doi.org/10.1016/j.jweia.2007.06.034 